BULL. AUSTRAL. MATH. SOC.

VOL. $14(1976), 417-423$.

\title{
Existence theory \\ for the complex nonlinear complementarity problem
}

\section{J. Parida and B. Sahoo}

The main result in this paper is an existence theorem for the following complex nonlinear complementarity problem: find $z$ such that

$$
\begin{gathered}
g(z) \in S^{*}, z \in S, \\
\operatorname{Re}(g(z), z)=0,
\end{gathered}
$$

where $S$ is a polyhedral cone in $C^{n}, S^{*}$ the polar cone, and $g$ is a mapping from $c^{n}$ into itself. It is shown that the above problem has a unique solution if the mapping $g$ is continuous and strongly monotone on the polyhedral cone $S$.

\section{Introduction}

In a recent paper [4], the authors have shown that if $g: c^{n}+c^{n}$ is continuous and monotone on the polyhedral cone $s \subset c^{n}$ and if there is a $u \in S$ with $g(u) \in$ int $S^{*}$, then the nonlinear complementarity problem

$$
\begin{gathered}
g(z) \in S^{*}, z \in S, \\
\operatorname{Re}(g(z), z)=0,
\end{gathered}
$$

has a solution. The solution is unique if $g$ is strictly monotone on $S$.

In this paper, we are concerned mainly with the problem of the existence and uniqueness of a solution to (1.1) under coercivity conditions

Received 25 February 1976. 
on the mapping $g$. It is shown that (I.I) has a unique solution if $g$ is continuous and strongly monotone on the polyhedral cone $s \subset C^{n}$. Further, assuming that $g$ is analytic on an open convex set containing $S$, some existence results have also been obtained by restricting the growth of the jacobian matrix $J_{g}(z)$ of $g$. Through our results, some well-known theorems proved by Mond [2] in the complex linear case, and Karamardian [1] and Moré [3] in the real case are carried over to nonlinear mappings in complex space.

\section{Notations and definitions}

Let $c^{n}\left[R^{n}\right]$ denote the $n$-dimensional complex [real] space, with hermitian [euclidean] norm. $R_{+}^{n}=\left\{x \in R^{n}: x_{i} \geq 0,1 \leq i \leq n\right\}$ denotes the non-negative orthant of $R^{n}$. If $A$ is a complex matrix or vector, then $A^{T}, \bar{A}$, and $A^{H}$ denote its transpose, complex conjugate, and conjugate transpose. For $x, y \in C^{n},(x, y)=y^{H} x$ denotes the inner product of $x$ and $y$. For an analytic mapping $g: C^{n} \rightarrow C^{n}, J_{g}(z)$ denotes the jacobian matrix of $g$ at $\boldsymbol{z}$.

A nonempty set $S \subset C^{n}$ is a polyhedral cone if for some positive integer $k$ and $A \in C^{n \times k}$,

$$
S=\left\{A x: x \in R_{+}^{k}\right\} .
$$

The polar of $S$ is the cone $S^{*}$ defined by

$$
S^{*}=\left\{y \in C^{n}: x \in S \Rightarrow \operatorname{Re}(x, y) \geq 0\right\} \text {. }
$$

Let $S$ be a polyhedral cone in $c^{n}$. For a scalar $p>0$, we denote $z(p)=\left\{A x: x_{i}=p, 1 \leq i \leq k\right\}$, where $A$ and $k$ determine the cone $S$. For any $z=A x \in S$, we write $z \leq z(p)$ if $\|x\|_{\infty} \leq p$, and $z<z(p)$ if $\|x\|_{\infty}<p$, where $\|x\|_{\infty}=\max \left\{\left|x_{i}\right|: 1 \leq i \leq k\right\}$.

A mapping $g: c^{n} \rightarrow c^{n}$ is said to be monotone on $S$ if $\operatorname{Re}(g(x)-g(y), x-y) \geq 0$ for each $x, y \in S$, and strictly monotone if the 
strict inequality holds whenever $x \neq y$.

A mapping $g: c^{n} \rightarrow c^{n}$ is said to be strongly $S$-monotone if there exists a scalar $c>0$ such that, for all $x, y \in S$ with $x-y \in S$,

$$
\operatorname{Re}(g(x)-g(y), x-y) \geq c\|x-y\|^{2},
$$

where $\|\cdot\|$ denotes the hermitian norm. It is said to be strongly monotone on $S$ if (A) holds for all $x$ and $y$ in $S$.

An $n \times n$ matrix $M(z)$, whose elements $m_{i j}(z)$ are functions defined on $S \subset C^{n}$, is said to be strongly positive definite on $S$ if there exists a scalar $c>0$ such that, for every $z \in S$,

$$
\operatorname{Re}\left(y^{H} M(z) y\right) \geq c\|y\|^{2}
$$

for all $y \in c^{n}$.

3. An existence theorem for the nonlinear complementarity problem We shall make use of the following results obtained in [4].

THEOREM 3.1. Let $g: c^{n}+c^{n}$ be a continuous mapping on the polyhedral cone $s \subset c^{n}$. If there are vectors $z(p), u \in S$, with $u<z(p)$ such that $\operatorname{Re}(g(z), z-u) \geq 0$ for $a z z \quad z=A x$ in $S$ with $\|x\|_{\infty}=p$, then there is a $z^{0} \leq z(p)$ in $S$ with $\operatorname{Re}\left(g\left(z^{0}\right), z-z^{0}\right) \geq 0$ for all $z \in S$.

LEMMA 3.2. Let $S$ be a polyhedral cone in $c^{n}$, and let $g: c^{n} \rightarrow C^{n}$ be continuous on $S$. If there is a $z^{0} \in S$ such that $\operatorname{Re}\left(g\left(z^{0}\right), z-z^{0}\right) \geq 0$ for all $z \in S$, then $z^{0}$ is a solution to (1.1).

We now prove a lemma which plays an important role in establishing our main existence and uniqueness theorem.

LEMMA 3.3. Let $g: c^{n} \rightarrow c^{n}$ be a continuous fionction on the polyhedral cone $s \subset C^{n}$. If there is a $u \in S$ with

$$
\lim _{\|z\|+\infty} \frac{\operatorname{Re}(g(z), z-u)}{\|z\|}=+\infty \text {, }
$$


then we can find a scalar $p>0$ such that $u<z(p)$, and for all $z=A x$ in $S$ with $\|x\|_{\infty}=p$,

$$
\operatorname{Re}(g(z), z-u)>0 \text {. }
$$

Proof. Let $u \in S$ satisfy (3.1). This implies that there is a vector $v \in S$ such that $\|v\|>\|u\|$, and $\operatorname{Re}(g(z), z-u)>0$ for all $z$ in $S$ with $\|z\| \geq\|v\|$. Since $S$ is a polyhedral cone, there exist a positive integer $k$ and a matrix $A \in C^{n \times k}$ such that $S=\left\{A x: x \in R_{+}^{k}\right\}$. If $v=A t, \quad t \in R_{+}^{k}$, set $\alpha=\|t\|_{\infty}$ and

$$
\beta=\min _{y \in V}\|y\| \text {, }
$$

where $V=\left\{A x \in S:\|x\|_{\infty}=\alpha\right\}$. Now choose $p=\alpha\|v\| / \beta$. By the definition of $p$, it is clear that $u<z(p)$, and for all $z=A x \in S$ with $\|x\|_{\infty}=p$, we have $\|z\| \geq\|v\|$. The result of the lemma is then obvious.

Now we are ready to establish the following existence and uniqueness theorem.

THEOREM 3.4. Let $g: c^{n} \rightarrow c^{n}$ be a continuous mapping on the polyhedral cone $S$, and assume that $g$ is strongly monotone on $S$. Then there is a unique solution to (1.1).

Proof. Since $g$ is strongly monotone on $S$, there is a scalar $c>0$ such that, for $x$ and $y$ in $S$,

$$
\operatorname{Re}(g(x)-g(y), x-y) \geq c\|x-y\|^{2} .
$$

If we set $y=0$ in (3.3), then we have

$$
\frac{\operatorname{Re}(g(x), x)}{\|x\|} \geq \frac{\operatorname{Re}(g(0), x)}{\|x\|}+c\|x\| .
$$

Since by the Schwarz inequality,

$$
\operatorname{Re}(g(0), x) \leq|(g(0), x)| \leq\|g(0)\|\|x\|,
$$

from (3.4) we obtain

$$
\frac{\operatorname{Re}(g(x), x)}{\|x\|} \geq c\|x\|-\|g(0)\|
$$

and consequently the hypothesis of Lemma 3.3 is satisfied by $g(z)$ with 
$u=0$. The existence of a solution to (1.1) then follows from Theorem 3.1 and Lemmes 3.2 and 3.3. Since strong monotonicity implies strict monotonicity, uniqueness follows from Remarks 4.3 of [4].

REMARKS 3.5. If $g(z)$ is defined by $g(z)=M z+q$ for some matrix $M$ and $q \in c^{n}$, then $g$ is strictly monotone on $S$ if $M$ is positive definite. Thus, strictly monotone functions are nonlinear versions of positive definite matrices. Although the uniqueness part of Theorem 6 of Mond [2] in the linear complementarity problem on positive definite matrices extends to the nonlinear strictly monotone functions, it is not known whether this is the case for the existence part. However, Theorem 3.4 extends these results to the nonlinear strongly monotone functions which are necessarily strictly monotone.

An $n \times n$ matrix $M$ is said to be strictly copositive with respect to $S \subset c^{n}$ if $\operatorname{Re}\left(z^{H} M z\right)>0$ for all $z \in S$. It is easy to see that if $g(z)=M z+b$, where $M$ is strictly copositive with respect to $S$, then $g$ satisfies (3.1) with $u=0$. In that case, the linear complementarity problem

$$
\begin{gathered}
z \in S, M z+b \in S^{*}, \\
\operatorname{Re}\left(z^{H}(M z+b)\right)=0
\end{gathered}
$$

possesses a solution, but uniqueness is not assured.

If $S=R_{+}^{n}$ and $g: R^{n} \rightarrow R^{n}$ is a continuous mapping on $R_{+}^{n}$, then Theorem 3.4 reduces to the result of Karamardian [1, Theorem 2.1]. In [3], Moré has extensively studied coercivity conditions in nonlinear complementarity problems in real $n$-space. It is to be noted that in this section an effort is made to extend some of his results to nonlinear mappings in complex space.

4. The nonlinear complementarity problem with positive definite jacobians In what follows, we shall always assume that $g: c^{n}+c^{n}$ is analytic on $C^{n}$ and that its jacobian matrix $J_{g}(z)$ is positive definite at some or all points $z \in C^{n}$.

We shall make use of the following two lemmas. 
LEMMA 4.1. Let $g: S \rightarrow C^{n}$ be analytic on $S$. Let $J_{g}(z)$ be its jacobian matrix at $z \in S$, and let $M(z)$ be the hermition part of $J_{g}(z)$. If $\alpha(z)$ is the smallest eigenvalue, which is necessarily real, of $M(z)$, then $J_{g}(z)$ is strongly positive definite on $S$ if, and only if, there exists a scalar $c>0$ such that $\alpha(z) \geq c$ for all $z \in S$.

Proof. Note that $J_{g}(z)$ is strongly positive definite if, and only if, $M(z)$ is strongly positive definite in the usual sense of the term. This follows since only the hermitian part of a complex matrix contributes to the real part of its quadratic form. It can be checked that a hermitian matrix is strongly positive definite if, and only if, its eigenvalues are positive and bounded below by a positive scalar. The assertion of the lemme follows by taking $c$ to be the least of all such lower bounds as $z$ varies over the whole of $S$.

LEMMA 4.2. Let $g: K \subset c^{n} \rightarrow c^{n}$ be conalytic on the open convex set $K$. Let $J_{g}(z)$ be its Jacobian matrix at $z \in K$. Then $g$ is strongly monotone on $K$ if $J_{g}(z)$ is strongly positive definite on $K$.

Proof. Consider the scalar function $\varphi:[0,1] \rightarrow c^{l}$ defined as

$$
\varphi(\lambda)=(x-y)^{H} g(\lambda x+(1-\lambda) y) .
$$

From (4.1) and the analyticity of $g$, we have

$$
\begin{gathered}
\varphi(1)-\varphi(0)=(x-y)^{H}(g(x)-g(y)), \\
\varphi^{\prime}(\lambda)=(x-y)^{H} J_{g}(\lambda x+(1-\lambda) y)(x-y) .
\end{gathered}
$$

Since $K$ is convex, $x, y \in K$ implies that $\lambda x+(1-\lambda) y \in K$ for all $0 \leq \lambda \leq 1$. From the strong positive definiteness of $J_{g}(z)$ on $K$, we then have

$$
\operatorname{Re}(x-y)^{H} J_{g}(\lambda x+(1-\lambda) y)(x-y) \geq c\|x-y\|^{2}
$$

for all $x, y \in K$ and all $0 \leq \lambda \leq 1$. But 
(4.5) $\quad \int_{0}^{1} \operatorname{Re} \varphi^{\prime}(\lambda) d \lambda=\operatorname{Re}(\varphi(1)-\varphi(0))$.

Finally, from (4.2)-(4.5), it follows that $g$ is strongly monotone on $K$.

THEOREM 4.3. Let $g: K \subset c^{n} \rightarrow c^{n}$ be analytic on the open convex set $K \supset S$. If all the eigenvalues of the hermitian part of the jacobian matrix $J_{g}(z)$ of $g$ are bounded below by a positive scalar for all

$z \in S$, then there exists a unique solution to (1.1).

Proof. The result follows from Theorem 3.4 and Lemas 4.1 and 4.2 .

\section{References}

[1] S. Karamardian, "The nonlinear complementarity problem with applications, part 1", J. Optimization Theory App Z. 4 (1969), $87-98$.

[2] Bertram Mond, "On the complex complementarity problem", Buzl. Austraz. Math. Soc. 9 (1973), 249-257.

[3] Jorge J. Moré, "Coercivity conditions in nonlinear complementarity problems", SIAM Rev. 16 (1974), I-16.

[4] J. Parida and B. Sahoo, "On the complex nonlinear complementarity problem", Bull. Austral. Math. Soc. 14 (1976), 129-136.

Department of Mathematics,

Regional Engineering College,

Rourkela,

Orissa,

India. 